Documentation et bibliothèques

DOCUMENTATION BIBLIOTHËQUES

\title{
Pour un engagement socio-politique des bibliothécaires québécois
}

\section{Georges Cartier}

Volume 19, numéro 3, septembre 1973

URI : https://id.erudit.org/iderudit/1055796ar

DOI : https://doi.org/10.7202/1055796ar

Aller au sommaire du numéro

\section{Éditeur(s)}

Association pour l'avancement des sciences et des techniques de la documentation (ASTED)

\section{ISSN}

0315-2340 (imprimé)

2291-8949 (numérique)

Découvrir la revue

\section{Citer cet article}

Cartier, G. (1973). Pour un engagement socio-politique des bibliothécaires québécois. Documentation et bibliothèques, 19(3), 101-106.

https://doi.org/10.7202/1055796ar

\section{Résumé de l'article}

Face aux problèmes d'ordre socio-politique, le niveau de tolérance des bibliothécaires québécois est élevé. Quelles en sont les causes déterminantes : comportement de colonisé, intégration au milieu social insuffisante, éthique professionnelle, sauvegarde d'intérêts financiers, droit d'expression limité, préoccupations internationales plutôt que nationales ? Pourquoi les bibliothécaires devraient-ils s'engager ? Collectivement! Pourquoi au québec plus qu'ailleurs? Que peut y gagner la profession?

Autant de questions que pose cet article et auxquelles chacun est libre d'apporter sa propre réponse, même si l'auteur y invite les membres de la profession à une engagement socio-politique collectif, à l'intérieur des cadres de leur association nationale.
Tous droits réservés ( $)$ Association pour l'avancement des sciences et des techniques de la documentation (ASTED), 1973
Ce document est protégé par la loi sur le droit d'auteur. L'utilisation des services d'Érudit (y compris la reproduction) est assujettie à sa politique d'utilisation que vous pouvez consulter en ligne.

https://apropos.erudit.org/fr/usagers/politique-dutilisation/ 


\section{Pour un engagement socio-politique des bibliothécaires québécois ${ }^{1}$}

\author{
Georges Cartier \\ Ecole de bibliothéconomie \\ Université de Montréal
}

Face aux problemes d'ordre sociopolitique, le niveau de tolérance des bibliothécaires québécois est élevé. Quelles en sont les causes déterminantes: comportement de colonisé, intégration au milieu social insuffisante, éthique professionnelle, sauvegarde d'intérêts financiers, droit d'expression limité, préoccupations internationales plutôt que nationales? Pourquoi les bibliothécaires devraient-ils s'engager? Collectivement! Pourquoi au québec plus qu'ailleurs? Que peut y gagner la profession?

Autant de questions que pose cet article et auxquelles chacun est libre d'apporter sa propre réponse, même si l'auteur y invite les membres de la profession à une engagement socio-politique collectif, à l'intérieur des cadres de leur association nationale.

Toute personne a droit à la liberté de pensée, de conscience, d'opinion et d'expression.

Ce droit implique la liberté d'adopter et de manifester, pacifiquement, ses convictions et ses idées, individuellement ou collectivement, tant en public qu'en privé.

(Charte québécoise des droits de I'homme, projet soumis à la collectivité et à l'Etat québécois par la Ligue des droits de l'homme, au mois de mai 1973)

En 1965, l'ex-directeur de la Bibliothèque

\footnotetext{
1. Dans la livraison de décembre 1972 (vol. XVII, no 4) du Bulletin de I'ACBLF, Laurent-G. Denis a publié un article intitulé: "Engagement socio-politique des bibliothécaires américains et canadiens-anglais" Très documenté, ce texte nous apportait sur ce sujet une intéressante information et de nombreuses références.

Délibérément, j'ai choisi une approche toute différente. celle d'une réflexion libre sur cette question controversée, dont le but évident n'est autre que de susciter une interro gation chez les membres de notre profession.
}

de la Législature, Jean-Charles Bonenfant, disait aux membres de l'ACBLF: "Notre profession doit faire de nous les gens les plus tolérants du monde, car les livres et les publications variées que nous conservons nous ont depuis longtemps enseigné que la vérité est ondoyante et diverse." 2 Ce souhait s'est en quelque sorte réalisé, et même au-delà des limites qu'entrevoyait sans doute le conférencier. En effet, collectivement, les bibliothécaires me paraissent fort tolérants; et dans certains cas, nous serions portés à croire qu'ils le sont trop. Je n'ignore pas les luttes qu'ils ont livrées et continuent de soutenir pour vaincre l'état de pauvreté d'un grand nombre de leurs bibliothèques. Par exemple, contre la maigreur des budgets, ils n'ont cessé de revendiquer des crédits accrus; contre l'exiguiité des locaux, des espaces supplémentaires; contre le manque de personnel pour assurer des services adéquats, des postes de plus en plus nombreux. Ils n'hésitent plus autant qu'autrefois à protester énergiquement contre certains gestes de nos dirigeants. Ils les discutent, présentent des propositions nouvelles, différentes, et les défendent avec acharnement parfois et d'une façon de plus en plus structurée, efficace, exigeant des rencontres avec les autorités, soumettant des mémoires de plus en plus fouillés, réclamant des réponses claires et précises.

En combien d'occasions, ces dernières années, notre association a-t-elle eu à se prononcer fermement, à adopter des lignes de conduite souvent pénibles à élaborer et surtout difficiles à défendre! Elle l'a fait avec un dynamisme et un courage, n'hésitons pas à le dire, souvent admirables. D'autant plus qu'aucun des membres de notre profession n'a jamais sacrifié une partie de ses loisirs à la défense de ces causes pour une quelconque rétribution. Nous sommes encore idéalistes; tant mieux, en un certain sens! Les groupes sociaux se corrompent toujours suffisamment vite.

Tout récement, l'Affaire des manuscrits en a été une autre preuve. Si je me permets de citer cet exemple, c'est qu'il m'a permis au plus haut point de vérifier personnellement la vérité de mes assertions. Pour une telle question, de tous les coins du Québec, s'est levée la profession, dans un grand mouvement de solidarité. Associations et groupes divers ont discuté, adopté des résolutions, préparé des

\footnotetext{
2. Jean-Charles Bonenfant, "Le bibliothécaire canadienfrançais et les Canadiens anglais", in Le bibliothécaire de langue française dans la société canadienne; rapports des travaux du 21e congrès de l'ACBLF, tenu à Ottawa, du 8 au 11 octobre 1965. Montréal, ACBLF, 1966, 115.
} 
contre-propositions; lettres et documents divers ont envahi le ministère des Affaires culturelles et les salles de rédaction des journaux. Spontanément, toute la profession s'était engagée dans cette lutte, une lutte qui pourtant n'affectait directement qu'une seule bibliothèque. Du même coup, ce geste unanime manifestait l'importance que les bibliothécaires attachaient à leur Bibliothèque nationale.

Mais ce n'est qu'un cas, et combien d'autres exemples n'y aurait-il pas à citer. Sur un plan strictement professionnel, l'ACBLF a donc adopté des positions fermes, courageuses à plusieurs occasions, les rendant publiques et les défendant de façon soutenue. Cependant, n'a-t-elle pas rigoureusement limité ses interventions au domaine bibliothéconomique, même s'il s'est imposé à plusieurs reprises qu'elle élargisse son champ d'action et qu'elle démontre un plus haut degré d'intégration au milieu québécois en prenant officiellement position sur des questions socio-politiques?

N'y a-t-il pas eu, au Québec, le célèbre Bill 63 qu'on ne peut encore qualifier "de triste mémoire'i, puisqu'il est bien vivant et se porte mieux qu'à sa naissance, assimilant chaque jour aux anglophones quelques citoyens de plus, ce qui fait forcément, mathématiquement, que l'avenir du Québec est délesté, toutes les vingt-quatre heures, d'un nombre égal de francophones? Aimerions-nous mieux, nous aussi, planifier nos lendemains québécois avec une rigueur tout aussi cynique, préférant en définitive diriger un jour des bibliothèques de langue anglaise? D'ailleurs, cette évolution est amorcée, si nous en jugeons par les collections d'un grand nombre de nos bibliothèques supposément de langue française; dans certains cas, elle est même si avancée, qu'une bibliothèque spécialisée, située dans une région entièrement francophone et exclusivement utilisée, je crois, par des francophones, a jugé nécessaire de constituer son fichier par matières en langue anglaise.

Egalement, n'avons-nous pas connu, au Québec, la Crise d'Octobre '70, la restriction des libertés civiles, la répression policière, la lutte du Front commun, l'emprisonnement des trois chefs de centrales syndicales, la déclassification des enseignants, et même l'intrusion fréquente de forces policières dans une bibliothèque? N'y a-t-il pas eu ce combat de David et Goliath qu'a été la lutte du Cegep Saint-Laurent avec le ministère de l'Education? Et je doute, au moment d'écrire cet article, que le dénouement final soit celui de la Bible!
De tout cela, et de multiples autres questions socio-politiques, les bibliothécaires parlent entre eux, c'est certain. Mais dépouillez les archives de l'association, consultez les résolutions du Conseil, les voeux des assemblées générales: vous n'en trouverez mot, ou quasi. Il est révélateur de constater que les bibliothécaires, lors de plusieurs congrès de l'ACBLF consacrés précisément à des aspects sociaux, ont à peine effleuré le thème qui leur était proposé. A lire les actes des congrès de 1957, 1958 et 1965 , on ne peut que déduire que la profession n'était pas encore prête à aborder de tels sujets. La formulation des thèmes invitait à des débats passionnés, incitait à des prises de position éclatantes, pour ne pas dire fracassantes: $L a$ bibliothèque dans le milieu social, Bibliothèque et vie nationale, Le bibliothécaire de langue française dans la société canadienne. Au lieu d'aborder de front ces questions fondamentales, les bibliothécaires se sont retranchés derrière les vieux problèmes, plus rassurants, de la classification, du catalogage, du choix des livres, et autres activités de ce genre, très bibliothéconomiques. . .

Personnellement, je conçois mal que notre profession puisse être témoin des profonds et larges remous qui se produisent dans notre société sans en manifester une certaine prise de conscience collective. Peu de pays sont aujourd'hui aussi propices à un engagement socio-politique que le Québec. Les bouleversements qui le transforment depuis plus de dix ans nous secouent sans répit. Nous sommes littéralement assaillis, en tant que citoyens québécois, et même notre volonté de quiétude la plus déterminée, qui nous incite périodiquement à nous retirer dans le silence, est sans cesse violée, jusque dans ses derniers retranchements. La poussée des événements, par vagues successives, ne peut forcément que nous entraîner, il me semble, à lutter contre ou avec ces forces d'innovation ou d'érosion, dont le ressac est souvent plus dangeureux que le choc initial. Néanmoins, tout se passe comme si nous avions décidé, nous bibliothécaires, de ne pas participer. Comme si nous avions choisi de nous laisser balloter au gré des courants. Oui, nous sommes tolérants. . jusqu'à nous satisfaire de lorgner par une fenêtre le défilé des turpitudes qui font se lever des groupes importants de citoyens. Alors qu'eux témoignent hautement, violemment parfois, leur désapprobation, nous, nous tolérons même notre tolérance!

Oh! je sais bien qu'individuellement, nous nous insurgeons ou nous réjouissons, que nous crions au scandale ou applaudissons; nous réagissons, quoi! Du moins, je l'espère. 
Mais collectivement, en tant que profession, ne prenons-nous pas garde de nous impliquer dans quelque problème d'ordre social ou politique? Un peu trop, même! N'avons-nous pas écouté trop fidèlement la voix de celui qui nous rappellait, très justement par ailleurs, que "la vérité est ondoyante et diverse"? $\mathrm{Ne}$ sommes-nous pas trop tolérants lorsque nous acceptons sans mot dire l'usage de l'anglais dans des réunions de dix à quinze personnes, dont deux ou trois seulement sont de langue anglaise? Une telle soumission conduit à des situations aussi incongrues que celle où l'on peut observer des bibliothécaires francophones continuant à discuter entre eux en anglais, alors que leurs quelques collègues anglophones se sont retirés du dialogue depuis longtemps. Pour ma part, j'ai été très souvent témoin de telles scènes, et j'avoue en avoir éprouvé chaque fois une certaine gêne, pour ne pas dire une certaine honte face à semblable démission. Devant de telles inepties, alors qu'une si faible représentation du conquérant réussissait à imposer sa langue, j'avais du mal à ne pas croire, profondément et sincèrement, que nous ne méritons pas le qualificatif de colonisés.

Dans le domaine des bibliothèques publiques, pour ne citer que celui-là, si nous scrutons quelque peu la nature des collections de certaines bibliothèques, si nous évaluons la proportion des livres de langue anglaise et de langue française comparativement aux pourcentages respectifs de la population desservie, nous nous réservons, là encore, des surprises désagréables, qui nous indiquent clairement à quel point notre niveau de tolérance est élevé. Et que dire de cas particuliers de discrimination, outrageants, dans la répartition des subventions? La liste n'est certes pas inépuisable, mais elle se renouvelle et s'accroît sans cesse, chaque jour apportant son lot d'inédit ancien, maintenant un système d'injustice toléré depuis fort longtemps, depuis trop longtemps.

C'est pourquoi je m'explique mal ou plutôt j'accepte mal notre retranchement, pour ne pas dire notre retraite. Car, qu'on le veuille ou non, même si on réussit à maintenir un refus d'engagement, on ne peut échapper, il me semble, à l'intégration à son milieu social. II est aujourd'hui suffisamment prouvé que l'individu est moins fort que la société qui l'entoure et qui, par suite, le façonne. Pas plus que les autres le bibliothécaire n'échappe à cette règle. S'il se contente de s'y soumettre, il adopte une attitude essentiellement passive, qui le contraint vite à subir les événements au lieu d'y participer et de tenter, malgré tout, selon la mesure de ses moyens, d'en modifier le cours. A cette position néga- tive, ne faut-il pas préférer un engagement réfléchi, qui corresponde à une étape plus évoluée d'une intégration inévitable au milieu social, et combien plus dynamique? D'ailleurs, ne pourrait-on pas définir simplement l'engagement comme une intégration active au milieu social?

Le rôle que remplit tout bibliothécaire à l'intérieur de sa bibliothèque, dans ses relations avec la clientèle, que ce soit dans la catégorie des bibliothèques universitaires, publiques, spécialisées ou autres, devrait logiquement le conduire à des activités sociales nombreuses, diversifiées. Pourquoi parvenons-nous si mal ou si peu à élargir nos préoccupations en tant que membres d'une profession qui, précisément, doit de plus en plus s'affirmer, sur tous les plans, si elle désire vraiment se faire reconnaître et, comme toutes les autres, occuper pleinement sa place dans la société? D'autres professions ont même atteint le stade de l'engagement politique, depuis longtemps. On retrouve certains de leurs membres à l'Assemblée nationale et même au Conseil des ministres. A ma connaissance, on n'y a jamais encore vu un bibliothécaire, dont la présence, sans être indispensable, ne serait certes pas inutile, ne serait-ce que pour référer le Cabinet, lors de discussions souvent mal préparées sur des questions pourtant importantes, à des décisions antérieures et des arrêtés-en-conseil oubliés, faute d'avoir été bien classés!

II faut nous réjouir par contre que notre profession compte quelques bibliothécaires qui ont osé poursuivre des activités politiques, soit sur le plan municipal, soit au niveau provincial; et je sais que plusieurs militent aujourd'hui à l'intérieur de formations politiques. Mais ces actions individuelles, fort louables, sont-elles suffisantes pour que, en tant que groupe, nous ayons le droit de demeurer en marge? Comme si la question des affaires de la société et de l'Etat, par conséquent la politique, ne nous concernait pas?

Que craint-on, en fait? Serait-ce la menace du pouvoir qui dispense les crédits aux bibliothèques et les subventions à l'association? $Y$ aurait-il, à la source de notre mutisme, cette forme odieuse de baillonnement? Peut-être, bien que je me résigne mal à justifier ainsi nos craintes. D'ailleurs, sur quelle expérience financièrement néfaste seraient-elles fondées? Je n'en connais pas qui soit probante.

II est vrai que les bibliothécaires sont presque tous, d'une certaine façon, des fonctionnaires, que ce soit au niveau municipal ou provincial, scolaire ou universitaire. Mais être rétribués pour notre travail ne nous impose 
aucunement d'être à la solde de nos employeurs, au sens péjoratif. Bien que notre liberté de parole soit parfois plus restreinte que pour d'autres, elle n'en demeure pas moins aussi réelle et utilisable, notre marge de manoeuvres permises étant suffisante. Et si une certaine prudence doit toujours guider nos actes, elle ne doit tout de même pas nous paralyser complètement. La manière de poser un geste compte autant que l'acte même, très souvent, à tel point que les meilleures intentions, on le sait bien, sont souvent compromises par un manque de savoir-faire; et celui qui manie l'art réussit mieux, fréquemment, que celui qui possède la science; cela aussi, tout le monde le sait. C'est là une vérité, qu'on la trouve regrettable ou pas, qui prend toute son importance dans l'engagement social et politique d'une profession.

Certains ont prétendu que les Québécois étaient surtout menés par la peur. Ils n'ont peut-être pas eu tort, ni complètement, ni en tout temps. Il est évident que nous craignons toujours de nous engager publiquement, et certaines voix réussissent habituellement à faire taire celles qui proposent courageusement de poser des actes. N'avons-nous pas tendance à ériger en système le non-engagement, à faire de cette position tranquille la règle d'or de notre bien-être? Voilà peut-être jusqu'à présent notre forme d'engagement collectif face aux problèmes de l'heure les plus cruciaux: l'engagement dans le refus face aux remous du milieu. En somme, la solution de facilité par excellence!

En quelque sorte, nous sommes victimes d'un repliement collectif assez triste et difficilement conciliable avec le rôle d'intermédiaire, de documentaliste, qu'est celui du bibliothécaire.

Je n'ignore pas combien il est pénible, certains jours, de ressentir une lassitude énormément lourde, et combien il est difficile de la vaincre! II s'agit là d'une manifestation normale, très humaine, conséquente à l'épuisement qu'entraîne la succession des combats à livrer. On en arrive même à éprouver, à certaines heures, un dégoût profond, face au déséquilibre évident des partis en lice et des moyens utilisés. Une telle réaction me paraît non seulement justifiée et par suite fort compréhensible, mais aussi très saine. II n'en demeure pas moins qu'elle doit rester passagère, sa permanence risquant de constituer le début d'un cas inquiétant, qui est peut-être le nôtre. Je ne ferai jamais grief à qui que ce soit de ne pas se baigner dans une eau trop froide, mais je lui tiendrais sans doute rigueur de ne pas tenter de sauver quelqu'un en perdition pour cette seule raison égoïste.
Aussi paradoxal que cela paraisse, nous n'hésitons pas à nous engager, même politiquement, défendant nos opinions avec fermeté et violence parfois, dès que nous sommes en pays étranger. Que le Québec soit attaqué ou simplement critiqué par un étranger, alors que nous sommes dans son pays, et nous voilà lancés dans une opposition farouche, immédiate, quasi instinctive. Qui n'a pas fait l'expérience de ce réflexe d'auto-défense, qui révèle clairement une intégration profonde au milieu? Intégration qui, dans ces cas, va jusqu'à provoquer un engagement automatique, souvent agressif, par exemple sur le plan linguistique, dans les relations entre Français et Québécois.

Par cortre, les problèmes les plus vitaux du pays de séjour ne feront toujours qu'éveiller en nous un intérêt superficiel, même s'il semble très vif, car ils n'atteignent pas les couches émotives de notre personnalité et n'engagent ni notre présent, ni notre avenir. Les difficultés d'Allende, l'état des hôpitaux de Paris, l'holocauste des bonzes vietnamiens, la guerre du Biafra, la lutte de l'Irlande, l'affaire du Watergate et les mille nouvelles quotidiennes que nous apportent les média modernes de communication, nous touchent certainement, nous ébranlent même, mais il ne s'agit presque toujours que de légères secousses sismiques, qui ne nous mettent aucunement en marche pour nous porter à la défense d'une cause suprême. Intérieurement, le temps des Croisades est révolu, et nous assistons en spectateurs aux bouleversements qui modifient chaque jour la face du monde. Souvent même, avouons-le, en spectateurs assez indifférents.

Mais au Québec, ne sommes-nous pas les acteurs du drame et l'angle de la scène ne doit-il pas modifier tout notre jeu? Que penserions-nous du bibliothécaire indifférent de l'Allemagne des années '30, de l'Etat d'Israël ou de l'Irlande d'aujourd'hui, de la Tchécoslovaquie du coup de Prague, de la Pologne de la domination russe? Des bibliothécaires américains se sont opposés à la guerre du Vietnam jusqu'à manifester ouvertement leurs convictions; ce geste, je le considère heureux, tout comme juste la décision du Conseil de direction de l'American Library Association, qui a ordonné à son directeur d'émettre un chèque de $\$ 200$ pour le "National United Committee to Free Angela Davis", à l'appui d'une résolution du SRRT (Social Responsibilities Round Table) ${ }^{3}$. Je souhaiterais même que devienne officielle au Québec

3. "SRRT \$200 for Angela Davis Approved by ALA Exec Board," Library Journal, vol. 96, no. 11 (June 1, 1971), 1913. 
la politique définie par le Conseil d'administration de la bibliothèque publique de Hartford, selon laquelle est non seulement approuvée, mais encore encouragée toute participation des membres du personnel à des activités politiques, hors des heures de travail et des locaux mêmes de la bibliothèque ${ }^{4}$. Les exemples à citer seraient nombreux. Si on parcourt Library Literature, on découvre des références comme celles-ci: "U.S. Library Associations Protest Czech Takeover", "Librarians' Group Back Humphrey", etc. La seule compilation des années 1967-69 recense cinq colonnes d'environ trente-cinq titres chacune, consacrés au problème de la liberté intellectuelle, dont l'ampleur est telle que je n'ai osé l'aborder dans cet article, même si de multiples aspects sont étroitement reliés à l'engagement socio-politique. Pour les années 1970-1971, on trouve une colonne d'articles consacrés aux seules activités politiques des bibliothécaires! Chez nous, nous n'en sommes encore qu'à la naissance d'un mouvement comparable.

J'ai eu la curiosité de relire le Code d'éthique de la Corporation des bibliothécaires professionnels du Québec pour vérifier si un article ou l'autre ne s'opposerait pas à l'orientation que je défends. J'en cite l'article 19: "En tant que corps professionnel, la Corporation peut cependant prendre position contre une idéologie qui, selon l'avis majoritaire de ses membres, est de nature à nuire au développement des bibliothèques et à l'avancement de la culture et de la civilisation". Ces derniers mots sont si vastes dans leur signification qu'ils peuvent justifier toute prise de position, sur quelque plan que ce soit. $\mathrm{Ce}$ n'est donc pas par un souci d'éthique professionnelle que nous ne nous mêlons pas aux grands mouvements qui se produisent périodiquement au Québec.

Je maintiens, quant à moi, que notre participation de plus en plus active à la vie sociopolitique, notre alliance éclairée avec des groupes de citoyens qui revendiquent, protestent et exigent de l'Etat des politiques précises dans divers domaines, qui présentent aux gouvernants des projets aussi sérieux qu'une charte des droits de l'homme, ne peut que servir la profession tout entière et contribuer autant, sinon plus que tout autre moyen, à la faire rapidement reconnaître. Nous nous plaignons fréquemment d'être méconnus et nous maintenons notre tendance à nous confiner, bien abrités, dans les citadelles et les monastères que constituent nos bibliothèques. Et nous nous étonnons de ne pas êtres recon-

4. "Hartford Public Library Encourages Politicking", Library Journal, vol. 93, no. 17 (September 1, 1968), 2950. nus! De ne pas projeter une image dont le reflet nous agréerait mieux que celle qui nous parvient de divers milieux. Jusqu'à quel point le miroir que forment les autres fausse-t-il la réalité? II serait judicieux de temps à autre de se poser sérieusement cette question, de scruter les traits de cette image.

Enfin, certains opposent aux préoccupations nationales la vocation internationale du bibliothécaire. Personnellement, je ne vois aucune contradiction entre ces centres d'intérêt; au contraire, ils me paraissent parfaitement complémentaires. On n'atteint pas à l'internationalisme autrement que par la coopération entre les nations. Cette vérité de La Palice réside dans l'étymologie même du terme: inter-nations. Si l'on veut signifier que le bibliothécaire doit seulement se préoccuper de questions internationales, je m'inscris en faux contre cette idéologie, car elle recèle une illusion qui relève d'une imagination enfantine. D'ailleurs, je n'ai jamais rencontré, jusqu'à ce jour, un authentique citoyen du monde. II faudra, préalablement, découvrir un autre monde et établir une coopération intermondiale. . La pensée internationale du bibliothécaire, non seulement je l'accepte, mais je suis disposé à en favoriser le développement par tous les moyens possibles. Surtout sur le plan de la coopération, et encore plus si elle signifie que le bibliothécaire doit toujours agir en toute liberté et avec le respect absolu de la liberté d'autrui, sans discrimination, ni de race, ni de religion, ni de sexe, ni de langue, ni d'opinion politique ou autre, etc. Mais là encore, rien ne s'oppose à son engagement socio-politique, c'est-à-dire à son intégration active au milieu naturel que représente.pour lui son pays.

Pour nous, bibliothécaires québécois, assez lucides et sincères, je l'espère, pour ne pas nous leurrer, notre milieu naturel, notre environnement socio-politique, pollué ou non, c'est avant tout le Québec. Que ceux qui songent à me contredire aillent travailler deux ans seulement, même moins, dans une ville de l'est, du centre ou de l'ouest du Canada; leur opposition sera moins farouche à leur retour. II ne nous est plus permis de répéter l'erreur d'aiguillage de nos prédécesseurs, car leurs prédictions les plus sereines se sont avérées fausses, par exemple dans le cas de la participation progressive des Québécois francophones à la "Canadian Library Association". Les conférenciers des anciens congrès de l'ACBLF prévoyaient une présence de plus en plus large des bibliothécaires de langue française au sein de la CLA; aussi affirmaient-ils sans hésitation que cette association compterait un jour deux sections très fortes, l'une de langue anglaise, l'autre de langue fran- 
çaise. L'évolution des idées a contrecarré leurs vues, on le constate aujourd'hui; maintenant, comme un pourcentage croissant de ses compatriotes, le jeune bibliothécaire québécois cherche avant tout à s'intégrer à son milieu culturel propre, qui est essentiellement le Québec, et c'est là, par suite, que son engagement socio-politique pourra s'exprimer entièrement.

Voilà notre véritable orientation, notre première démarche essentielle. La seconde, celle de la coopération avec l'extérieur, celle de l'internationalisme, suivra d'autant mieux que la première aura été assumée pleinement. C'est la voie la plus sûre, la meilleure garantie contre les regrettables et stériles frictions avec nos collègues du Canada anglais, qui ont durant si longtemps marqué nos relations et ralenti notre progression. Aujourd'hui, notre association n'a rien à envier à la CLA, au contraire! Elle peut même prendre une autre coudée d'avance, si elle n'hésite pas à s'engager avec courage et diplomatie dans une action socio-politique, chaque fois que les événements le commandent. C'est à nous qu'il appartient de la conduire vers ces horizons nouveaux, par un engagement individuel authentique, fidèle à nos plus profondes racines de bibliothécaires francophones et québécois.•

\section{Prévenez le vol}

Checkpoint est un système avantgardiste de sécurité électronique à état solide et en circuits imprimés.

Les pages de garde, les ex-libris ou les pochettes des livres présensibilisés imposent leur présence lorsqu'ils sortent entre deux écrans sentinelles.

II est en usage dans plus de bibliothèques publiques et d'enseignement supérieur et protège plus de livres que n'importe quel autre système, tant au Canada qu'aux Etats-Unis et à Puerto Rico. Pour plus de renseignements ou pour une visite à l'un de nos nombreux clients canadiens, appelez:

CHECKPOINT SYSTEMS, INC.

22 Springdale Road

Cherry Hill, New Jersey 08034

Telephone: (609) 424-3035

REPRESENTANT AU CANADA

Paul S. CUGNO 119, av. Chestnut

Pointe-Claire 720 (Québec) Canada

Téléphone: (514) 695-7367

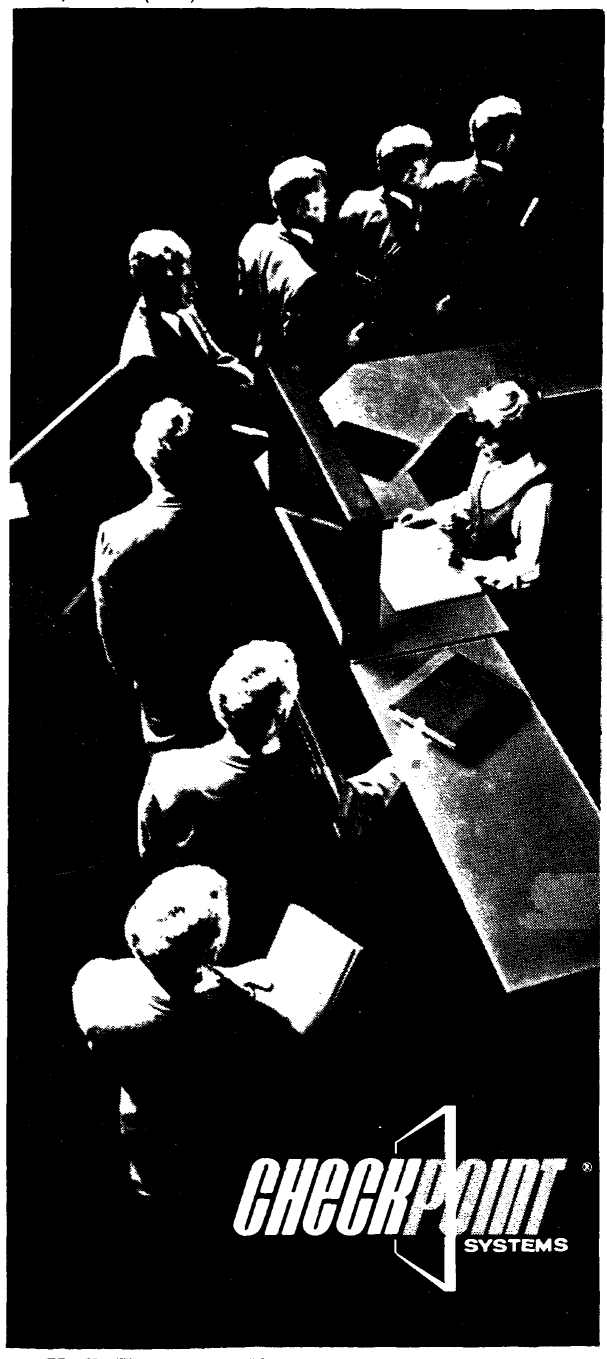

